\title{
Co-administration of aspirin and allogeneic adipose-derived stromal cells attenuates bone loss in ovariectomized rats through the anti-inflammatory and chemotactic abilities of aspirin
}

\author{
Hao Liu', Wei Li', Yunsong Liu², Xiao Zhang ${ }^{2}$ and Yongsheng Zhou ${ }^{2,3^{*}}$
}

\begin{abstract}
Introduction: Osteoporosis is a syndrome of excessive skeletal fragility characterized by the loss of mass and deterioration of microarchitecture in bone. Single use of aspirin or adipose-derived stromal cells (ASCs) has been recognized recently to be effective against osteoporosis. The goal of the study was to evaluate the osteogenic effects of the co-administration of aspirin and allogeneic rat adipose-derived stromal cells (rASCs) on ovariectomized (OVX)-induced bone loss in rats. The underlying mechanisms were investigated in vitro and in vivo.

Methods: Firstly, allogeneic rASCs were isolated and cultured, and the conditioned medium (CM) from the maintenance of rASCs was collected. Secondly, the OVX rats were administrated CM, rASCs, aspirin (ASP) or rASCS + ASP, respectively. Twelve weeks later, the anti-inflammatory and osteogenic effects were assessed by micro-CT, undecalcified histological sections, dynamic histomorphometric analyses and serologic assays for biochemical markers. Finally, a Transwell migration assay in vitro and cell-trafficking analyses in vivo were used to explore the effects of aspirin on rASC migration.

Results: Systemic administration of aspirin and rASCs attenuated OVX-induced bone loss better than single use of aspirin or ASCs ( $p<0.05$, respectively). Next, we analyzed the underlying mechanisms of the anti-inflammatory and chemotactic abilities of aspirin. Aspirin suppressed serum levels of the pro-inflammatory cytokines on tumor necrosis factor- $\alpha$ (TNF- $\alpha$ ) and interferon- $\gamma$ (IFN- - ), and the anti-inflammatory ability was positively associated with bone morphometry. Also, aspirin exhibited excellent chemotactic effects in vitro and accelerated the homing of allogeneic rASCs into bone marrow during early in vivo stages.
\end{abstract}

Conclusions: Co-administered aspirin and allogeneic ASCs can partially reverse OVX-induced bone loss in rats. This effect appears to be mediated by the anti-inflammatory and chemotactic abilities of aspirin.

Keywords: Allogeneic adipose-derived stromal cells, Aspirin, Osteoporosis, Bone regeneration, Cell homing

\footnotetext{
* Correspondence: kqzhouysh@hsc.pku.edu.cn

${ }^{2}$ Department of Prosthodontics, Peking University School and Hospital of Stomatology, 22 Zhongguancun South Avenue, Haidian District, Beijing 100081, China

${ }^{3}$ National Engineering Lab for Digital and Material Technology of Stomatology, Peking University School and Hospital of Stomatology, Beijing 100081, China

Full list of author information is available at the end of the article
}

\section{Biomed Central}

(c) 2015 Liu et al. Open Access This article is distributed under the terms of the Creative Commons Attribution 4.0 International License (http://creativecommons.org/licenses/by/4.0/), which permits unrestricted use, distribution, and reproduction in any medium, provided you give appropriate credit to the original author(s) and the source, provide a link to the Creative Commons license, and indicate if changes were made. The Creative Commons Public Domain Dedication waiver (http://creativecommons.org/publicdomain/zero/1.0/) applies to the data made available in this article, unless otherwise stated. 


\section{Introduction}

Osteoporosis is a syndrome of excessive skeletal fragility characterized by loss of the mass and deterioration of the microarchitecture of bone, which leads to fragility fractures [1]. The prevalence of osteoporosis has increased rapidly among the elderly as life expectancy has increased in industrialized countries [2].

Two major pharmacologic approaches to the treatment of osteoporosis are anabolic agents such as parathyroid hormone [3, 4] (which stimulate bone formation) or antiresorptive agents such as bisphosphonates, calcitonin, raloxifene, and estrogen (which inhibit bone resorption) $[5,6]$. These therapies have been shown to increase bone mineral density (BMD) and reduce the risk of fractures, but long-term safety and efficacy are ongoing concerns [7]. Thus, new safe and effective modalities are desired to ameliorate osteoporotic conditions.

Stem-cell therapy has been investigated for the repair and regeneration of damaged tissue in various conditions (e.g., myocardial ischemia [8, 9], stroke [10, 11], diabetes mellitus [12, 13], and cartilage/bone defects [14-16]) owing to the potential for multi-lineage differentiation of stem cells. Bone marrow (BM) has been most commonly used as a source of mesenchymal stem cells (MSCs), but the number and capacity for multi-lineage differentiation decline with age or health of donors [17-19]. Moreover, obtaining $\mathrm{BM}$ is an invasive procedure that can cause complications such as pain, bleeding, and infection.

To circumvent these limitations, adipose-derived stromal cells (ASCs) have been used recently as alternative sources of MSCs. ASCs have the following advantages over other sources of MSCs: multi-lineage MSCs have similar potential to that of bone marrow-derived mesenchymal stem cells (BMMSCs); ease of harvesting; and abundant sources [20-23]. Furthermore, by avoiding difficulties in obtaining sufficient numbers of autologous stem cells and decreased osteogenic potential of ASCs as a result of donor aging [24], ASCs from allogeneic donors could constitute a valuable alternative source of stem cells for therapeutic use because ASCs have superior immunomodulatory effects than other sources of MSCs. Taken together, ASCs may be a better source of stem cells for the treatment of osteoporosis $[25,26]$.

Aspirin is a widely used non-steroidal anti-inflammatory drug. Epidemiologic studies have suggested that aspirin might have a moderate beneficial effect on BMD in postmenopausal women [27]. Recently, Liu et al. reported that implantation of BMMSCs/hydrogel/aspirin resulted in improvement of bone generation in calvarial defects by improving the osteogenesis of, and by inhibiting the apoptosis of, BMMSCs [28]. Moreover, several lines of evidence have suggested that aspirin can inhibit osteoclastogenesis and improve osteogenesis by suppression of expression of pro-inflammatory cytokines, inhibition of activity of cyclooxygenase 1 and 2, and prostaglandin$\mathrm{E}_{2}$, and up-regulation of telomerase activity in BMMSCs [29-32]. Thus, we speculated that aspirin could favor stem cell-mediated bone regeneration by affecting multiple biological pathways.

Hu et al. demonstrated that aspirin can induce angiogenesis by promoting migration of endothelial progenitor cells [33]. Brunelli et al. also reported that HCT 1026 (a nitric oxide-donating agent with nonsteroidal anti-inflammatory activity) enhanced the therapeutic efficacy of arterially delivered donor stem cells by increasing their ability to migrate and reconstitute muscle fibers [34]. In the above-mentioned studies, aspirin or other nonsteroidal anti-inflammatory agents improved angiogenesis or reconstituted muscle fibers by promoting the migration of stem (progenitor) cells to the target organ. Therefore, we hypothesized that aspirin can improve osteogenesis (at least in part) by enhancing homing of stem cells into BM.

We wished to evaluate the effects of co-administration of aspirin and allogeneic rat adipose-derived stromal cells (rASCs) on ovariectomized (OVX)-induced bone loss in rats, and investigated the underlying mechanism of these effects in vitro and in vivo.

\section{Methods}

\section{Ethical approval of the study protocol}

All animal experiments were approved by the Animal Care and Use Committee of Peking University Health Science Center (approval number: LA2014233; Beijing, China).

\section{Isolation and maintenance of rASCs and preparation of conditioned medium}

All materials were purchased from Sigma-Aldrich (Saint Louis, MO, USA) unless stated otherwise. For rASCs, Lewis rats were euthanized at eight weeks of age by $\mathrm{CO}_{2}$ asphyxiation according to standard protocols. Visceral fat pads were minced and digested by type-I collagenase, filtered through cell strainers (pore size, $100 \mu \mathrm{m}$; Biologix, Lenexa, KS, USA) and centrifuged for 10 minutes at $380 \times g$ at room temperature. Cells were cultivated in maintenance medium [Dulbecco's modified Eagle's medium (DMEM) containing $10 \%$ fetal bovine serum (FBS), $100 \mathrm{U} / \mathrm{mL}$ penicillin $\mathrm{G}$, and $100 \mathrm{mg} / \mathrm{mL}$ streptomycin] at $37{ }^{\circ} \mathrm{C}$ in an atmosphere of $5 \% \mathrm{CO}_{2}$. rASCs of passage 3-5 were used and all cell-based experiments were repeated at least three times.

To prepare the conditioned medium (CM) of rASCs, cells were detached upon reaching $80 \%$ confluence in a $10-\mathrm{cm}^{2}$ dish and cultured for $12 \mathrm{~h}$ by DMEM containing $2 \%$ FBS. Next, rASCs were cultured by a serum-free DMEM for $12 \mathrm{~h}$ and the supernatant was collected and sterilized by passage through a filter (pore size, $0.22 \mu \mathrm{m}$ ). 


\section{Animals and administration procedure}

Rats were allowed free access to water and a maintenance diet in a 12-h light-dark cycle at a room temperature of $21 \pm 2{ }^{\circ} \mathrm{C}$. Rats were housed in groups of $\leq 5$.

Eight-week-old female F344 rats $(\mathrm{n}=70)$ were obtained from Vital River Inc. (Beijing, China). Bilateral OVX or sham operation was undertaken by standard methods under general anesthesia using pentobarbital sodium ( $50 \mathrm{mg} / \mathrm{kg}$, i.p.).

Four weeks after surgery, rats were assigned randomly into seven groups of ten: (1) sham rats were injected with vehicle (Sham group); (2) OVX rats were injected with vehicle (OVX); (3) OVX rats were injected with zoledronate (ZOL); (4) OVX rats were injected with allogeneic rASCs CM (ASC CM); (5) OVX rats were injected with allogeneic rASCs (ASC), (6) OVX rats were given a gavage of aspirin (ASP), (7) OVX rats were injected with rASCs and given a gavage of aspirin $(\mathrm{ASC}+\mathrm{ASP})$.

On days $1,2,8$, and $9,200 \mu \mathrm{L}$ of $\mathrm{CM}$ (total of $800 \mu \mathrm{L}$; ASC CM group) or $1.5 \times 10^{6}$ allogeneic rASCs in $200 \mu \mathrm{L}$ of DMEM (total of $6 \times 10^{6}$ cells; ASC and ASC + ASP groups) were injected into the lateral tail vein. Then, $100 \mathrm{mg} / \mathrm{kg}$ body weight of aspirin in $2 \mathrm{~mL}$ of physiologic $(0.9 \%)$ saline (1 \% DMSO assisted solubilization) was given by gavage every day of the total study period in ASP and ASC + ASP groups. In sham and OVX groups, DMEM and physiologic saline were administered each time. As a positive control, zoledronate $(100 \mu \mathrm{g} / \mathrm{kg}$, s.c.) was administered on day1 and day- 8 in the ZOL group.

Twelve weeks later, after euthanasia, the right tibia from each animal was dissected free from soft tissue thoroughly and fixed in $10 \%$ neutral-buffered formalin. Subsequently, it was used for micro-computed tomography $(\mathrm{CT})$ and histological slicing.

\section{Micro-CT analyses}

To evaluate the mass and microarchitecture in bone among the seven groups, micro-CT was undertaken using an Inveon MM system (Siemens, Munich, Germany). Images were acquired at an effective pixel size of $8.82 \mu \mathrm{m}$, voltage of $80 \mathrm{kV}$, current of $500 \mu \mathrm{A}$ and exposure time of $1,500 \mathrm{~ms}$ in each of the 360 rotational steps. Parameters were calculated using an Inveon Research Workplace (Siemens) as follows: bone volume/total volume (BV/TV), bone surface area/bone volume (BS/BV), trabecular thickness (Tb.Th), trabecular number (Tb.N), trabecular separation (Tb.Sp) and $\mathrm{BMD}$ in the trabecular region $(1-2 \mathrm{~mm}$ distal to the proximal epiphysis) according to guidelines set by the American Society for Bone and Mineral Research [35].

\section{Slicing of undecalcified bones and dynamic histomorphometric analyses}

Rats were injected with alizarin-3-methyliminodiacetic acid (30 mg/kg body weight, i.p.) ten days before euthanasia and calcein $(20 \mathrm{mg} / \mathrm{kg}$ body weight, i.p.) three days before. The tibia was fixed as described above and dehydrated in ethanol followed by embedding in destabilized methyl methacrylate resin. Next, sections were ground and polished to $40-60 \mu \mathrm{m}$ by an EXAKT precision cutting and grinding system (EXAKT Apparatebau, Hamburg, Germany) and stained (hemotoxylin and eosin). Dynamic histomorphometry was undertaken using Bioquant software (BioQuant, San Diego, CA, USA).

\section{Assay for biochemical markers}

For detection of biochemical markers of bone turnover and pro-inflammatory cytokines, blood samples were acquired from the aorta ventralis upon euthanasia. Serum was separated and stored at $-80{ }^{\circ} \mathrm{C}$. Serum levels of procollagen $1 \mathrm{~N}$-terminal peptide (P1NP), bone alkaline phosphatase (ALP), and tartrate-resistant acid phosphatase (TRAP) were measured using the relevant enzymelinked immunoassay (ELISA) kits (IDS, Frankfurt, Germany). Serum levels of calcium were measured using a Plasma Emission Spectrometer (iCAP 6000; Thermo Fisher Scientific, Waltham, MA, USA). Serum levels of tumor necrosis factor (TNF)- $\alpha$ and interferon (IFN) $-\gamma$ were measured using the relevant ELISA kits (eBioscience, San Diego, CA, USA). Samples were measured in duplicate (at least).

\section{Cell-migration assay in vitro and in vivo}

To explore the effects of aspirin on migration of rASCs, a transwell migration assay in vitro and cell-trafficking analyses in vivo were used.

For the transwell migration assay, $1 \times 10^{5}$ cells from passage 4 were loaded into the upper chamber of a 24-well transwell plate (pore size, $8 \mu \mathrm{m}$; Corning, Corning, NY, USA) and $600 \mu \mathrm{L}$ DMEM containing different concentrations of aspirin added to the lower chamber. Twenty-four hours later, cells on the upper surface of the membranes were removed with a cotton swab. Cells that had migrated to the lower surface of the membrane were fixed with $10 \%$ neutral-buffered formalin and stained for $15 \mathrm{~min}$ with $0.5 \%$ crystal violet. The number of cells that had migrated into the lower chamber was counted twice in five randomly selected microscopic fields (at $\times 400$ magnification) per filter by blind evaluations by two independent assessors. All aspirin concentrations were repeated at least three times.

For cell-trafficking analyses, a parallel assay using fluorescent dye-labeled cell injection was used. Forty 12-weekold F344 rats were divided into two groups of twenty animals four weeks after surgery: (1) OVX rats were given 
a gavage of vehicle (OVX-Vehicle); and (2) OVX rats were given a gavage of aspirin (OVX-ASP). A total of $100 \mathrm{mg} / \mathrm{kg}$ aspirin in $2 \mathrm{~mL}$ of physiologic saline was given by gavage on each day of the total study period. After one week of gavage, allogeneic rASCs were labeled with $5 \mu \mathrm{M}$ CM-DiL fluorescent dye (CellTracker ${ }^{\mathrm{Tm}}$ CM-DiL; Invitrogen, Carlsbad, CA, USA) according to the manufacturer's instructions for adherent cells. A total of $1.5 \times 10^{6} \mathrm{CM}-$ DIL-labeled allogeneic rASCs from Lewis rats in $200 \mu \mathrm{L}$ of DMEM were injected into F344 rats after four weeks of OVX $(n=4$, at each time point of then group). Rats were euthanized at $6,12,24,48$, and $72 \mathrm{~h}$ after cell injection. BM cells from the femur were obtained as described previously [36] and analyzed for CM-DiLpositive cells $\left(10^{4}\right.$ cells per sample) with LSRFortessa ${ }^{\text {su }}$ (BD Biosciences, Franklin Lakes, NJ, USA).

\section{Statistical analysis}

Data are shown as the mean \pm standard deviation. SPSS v16.0 (IBM, Armonk, NY, USA) was used for statistical analyses. Independent two-tailed Student's $t$-test or oneway analysis of variance (ANOVA) with post hoc least significant difference (LSD) were performed, and p-values less than 0.05 were considered significant.

\section{Results}

Radiologic and histologic evaluation of the mass and microarchitecture of bone

To assess the mass and microarchitecture of bone, BMD and bone morphometry were carried out using micro-CT (Fig. 1a) and the results are shown in Table 1. For BMD, micro-CT analyses of the proximal tibia ex vivo demonstrated that the OVX groups (OVX, ASC $\mathrm{CM}, \mathrm{ASC}$, ASP, and ASC + ASP groups, $\mathrm{n}=50$ ) exhibited significantly less BMD compared with the sham group except the ZOL group $(p<0.001)$. Interestingly, the BMD of the ASC CM group increased significantly compared with the OVX and ASC groups (OVX group $p=0.012$, ASC group $p=0.032$ ). However, inconsistent with BMD results, the ASC CM group showed a poor effect upon bone microarchitecture among the treatment groups (ASC CM, ASC, ASP, and ASC + ASP groups). The ASC CM group had a dramatically decreased BV/TV compared to the ASC + ASP group ( $p=$ $0.001)$ and a dramatically decreased $\mathrm{Tb}$.Th compared with the other treatment groups (ASC group $p=0.005$, ASP group $p=0.033$, ASC + ASP group $p<0.001)$. The ASC CM group had significantly increased BS/BV compared with the other treatment groups (ASC group, $p=$ 0.009; ASP group, $p=0.045$; ASC + ASP group, $p<0.001$ ). The ASC + ASP group had a significantly increased BV/ TV, Tb.N, and Tb.Sp compared with the ASC group and ASP group (for the ASC group and ASP group, respectively, BV/TV: $p=0.006$ and $p=0.002$; Tb.N: $p=0.006$ and $p=0.004$; Tb.Sp: $p=0.020$ and $p=0.013$ ).

To confirm radiologic evaluation of tibias, analyses of slices of hard tissues were undertaken and the results of bone morphometry from histology were similar to those of the micro-CT (data not shown) (Fig. 1b).

\section{Dynamic histomorphometric analyses}

Consistent with the analyses of bone microarchitecture, dynamic histomorphometric analyses also revealed significant increases in the bone formation rate (BFR) in

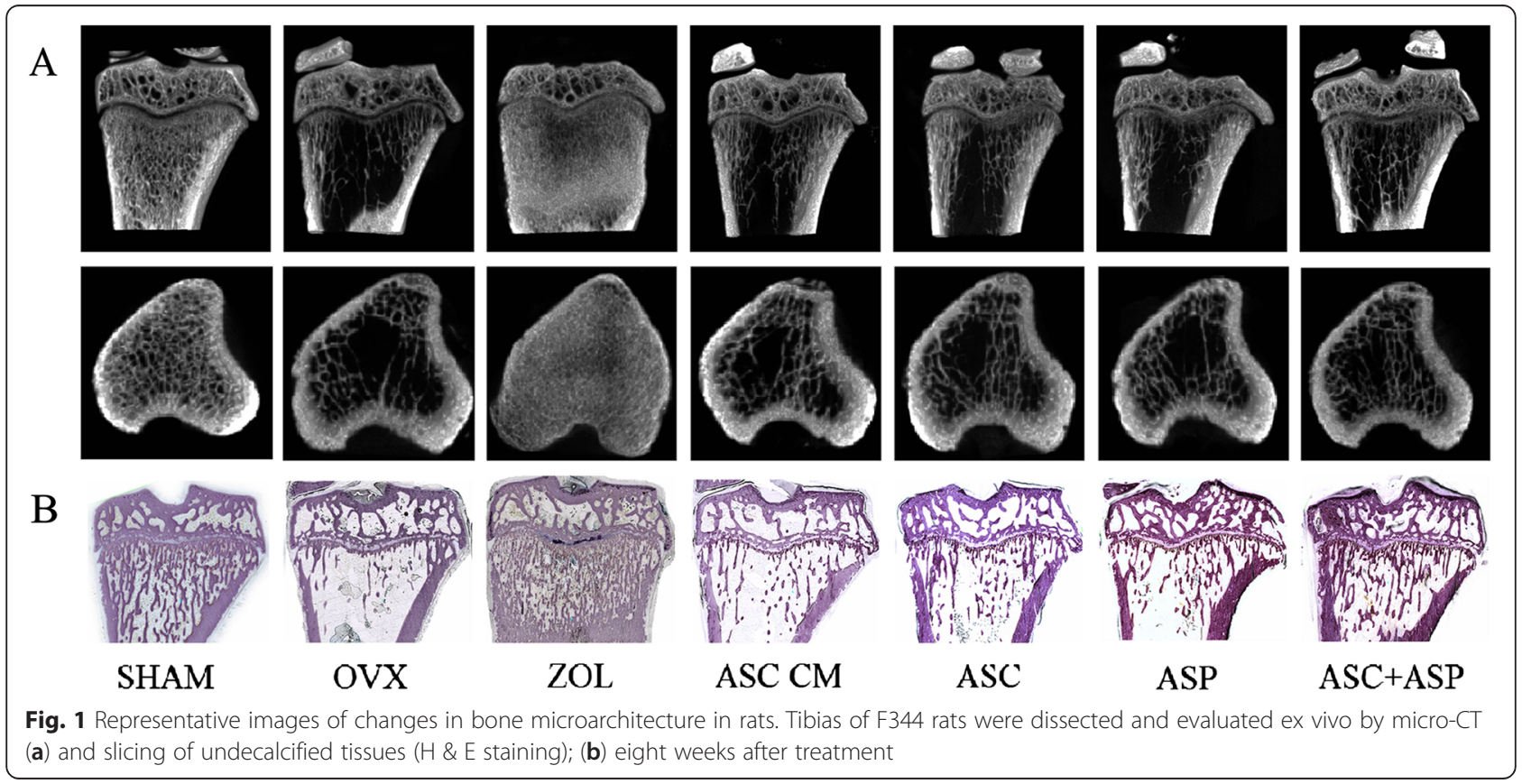


Table 1 Mineral density and histomorphometry of tibias in the seven groups of rats

\begin{tabular}{|c|c|c|c|c|c|c|c|}
\hline Groups & Sham & OVX & $\mathrm{ZOL}$ & ASC CM & ASC & ASP & \\
\hline$M D\left(\mathrm{~g} / \mathrm{cm}^{3}\right)$ & $1.791 \pm 0.048$ & $1.445 \pm 0.031^{a}$ & $1.969 \pm 0.069^{a, b}$ & $1.497 \pm 0.031 \mathrm{a}, \mathrm{b}, \mathrm{c}$ & $1.457 \pm 0.045^{a, c, d}$ & $1.466 \pm 0.034^{a, c}$ & $1.470 \pm 0.024^{a, c}$ \\
\hline V/TV (\%) & $44.009 \pm 2.432$ & $23.676 \pm 2.848^{\mathrm{a}}$ & $87.026 \pm 2.388^{a, b}$ & $29.216 \pm 2.435^{a, b, c}$ & $30.202 \pm 4.5699^{a, b, c}$ & $29.378 \pm 3.394^{a, b, c}$ & $34.521 \pm 1.965^{a, b, c, c, c}$ \\
\hline $\mathrm{S} / \mathrm{BV}\left(\mathrm{mm}^{-1}\right)$ & $33.857 \pm 2.247$ & $37.474 \pm 1.611^{\mathrm{a}}$ & $12.362 \pm 2.142^{a, b}$ & $35.788 \pm 1.108^{c}$ & $33.214 \pm 2.3722^{b, c, d}$ & $33.784 \pm 2.210^{b, c, d}$ & $32.162 \pm 1.642^{b, c, d}$ \\
\hline o.Th (mm) & $0.059 \pm 0.003$ & $0.053 \pm 0.002^{a}$ & $0.156 \pm 0.003^{a, b}$ & $0.056 \pm 0.002^{a, c}$ & $0.060 \pm 0.004^{b, c, d}$ & $0.059 \pm 0.004^{b, c, d}$ & $0.062 \pm 0.003^{b, c, d}$ \\
\hline & $7.376 \pm 0.204$ & & $8.008 \pm 0.252^{a, b}$ & $5.223 \pm 0.400^{a, b, c}$ & $4.979 \pm 0.540^{a, b, c}$ & $4.934 \pm 0.359^{a, b, c}$ & $5.551 \pm 0.410^{a, b, c, e, f}$ \\
\hline b.Sp (mm) & $0.077 \pm 0.008$ & $0.174 \pm 0.022^{a}$ & $0.026 \pm 0.007^{a, b}$ & $0.137 \pm 0.015^{a, b, c}$ & $0.143 \pm 0.025^{a, b, c}$ & $0.144 \pm 0.018^{a, b, c}$ & $0.119 \pm 0.012^{a, b, c, e, f}$ \\
\hline
\end{tabular}

Parameters of bone mineral density (BMD) and microarchitecture (BV/TV, BS/BV, Tb.Th, Tb.N, Tb.Sp) were measured in the trabecular bone of the proximal tibia (1-2 $\mathrm{mm}$ distal to the proximal physis) using micro-CT. Data are the mean $\pm S D, \mathrm{n}=70$

OVX ovariectomized, ZOL zoledronate, ASC adipose-derived stromal cells, CM conditioned medium, ASP aspirin, BV/TV bone volume/total volume, BS/BV bone surface area/bone volume, $T b . T h$ trabecular thickness, $T b . N$ trabecular number, $T b . S p$ trabecular separation

${ }^{\mathrm{a}} p<0.05$ vs. Sham, ${ }^{b} p<0.05$ vs. OVX, ${ }^{c} p<0.05$ vs. ZOL, ${ }^{d} p<0.05$ vs. ASC CM, ${ }^{e} p<0.05$ vs. ASC, ${ }^{f} p<0.05$ vs. ASP

the ASC + ASP group compared with the OVX, ASC $\mathrm{CM}$, and ASP groups (OVX group, $p<0.001$; ASP group, $p<0.001$; ASC CM group, $p=0.006$ ) (Fig. 2). However, the BFR of the ASP group was reduced significantly compared with the ASC group $(p=0.006)$. The BFR of the OVX groups (OVX, ASC CM, ASC, ASP, and ASC + ASP groups, $\mathrm{n}=50)$ was decreased significantly compared with the sham group $(p<0.001)$. The ZOL group showed irregular fluorescence and calculation of the BFR was difficult because osteoclast-directed bone resorption (which is usually coupled with bone formation) was inhibited (data not shown).

\section{Serological assessment of the formation and resorption of bone}

Data from the ELISA of P1NP, ALP, and TRAP from each group are shown in Fig. 3. As expected, serum levels of P1NP from the OVX groups (OVX, ZOL, ASC CM, ASC, ASP, and ASC + ASP groups, $\mathrm{n}=60$ ), a marker of bone formation, were significantly lower than those of the sham group (OVX, ZOL, ASC CM, and ASP groups, $p<0.001$; ASC group, $p=0.001$; ASC + ASP group, $p=0.011$ ). In accordance with bone histomorphometric analyses, serum levels of P1NP were dramatically higher in the ASC + ASP group than in OVX, ASC CM, and ASP groups (OVX group, $p<0.001$; ASC CM group, $p=0.027$; ASP group, $p=0.007$ ). Also, P1NP levels in the ASP group were decreased significantly compared with those of the ASC group ( $p=0.022$ ) (Fig. 3a).

Similar to the data from the ELISA for P1NP, serum levels of ALP from the OVX, ZOL, ASC, and ASP groups $(n=40)$ were significantly lower than those of the sham group (OVX group, $p=0.002$; ZOL group, $p<$ 0.001 ; ASC group, $p=0.018$; and ASP group, $p=0.003$ ). Moreover, ALP levels in the ASC + ASP group dramatically increased compared with the OVX, ZOL, and ASP groups (OVX group, $p=0.016$; ZOL group, $p=0.001$; ASP group, $p=0.018$ ) (Fig. 3b).

Moreover, serum levels of TRAP (a marker of bone resorption) were significantly lower in the sham group than in the OVX, ASC CM, ASC, ASP, and ASC + ASP groups (OVX, ASC CM, ASC and ASP groups, $p<0.001$; ASC + ASP group, $p=0.001)$. Notably, owing to the antiresorption effect of zoledronate in bone, TRAP levels in the ZOL group were decreased significantly compared with the OVX, ASC CM, ASC, and ASP groups (OVX, ASC CM, and ASP groups, $p<0.001$; ASC group, $p=$ 0.001). Serum levels of TRAP in the ASC + ASP group
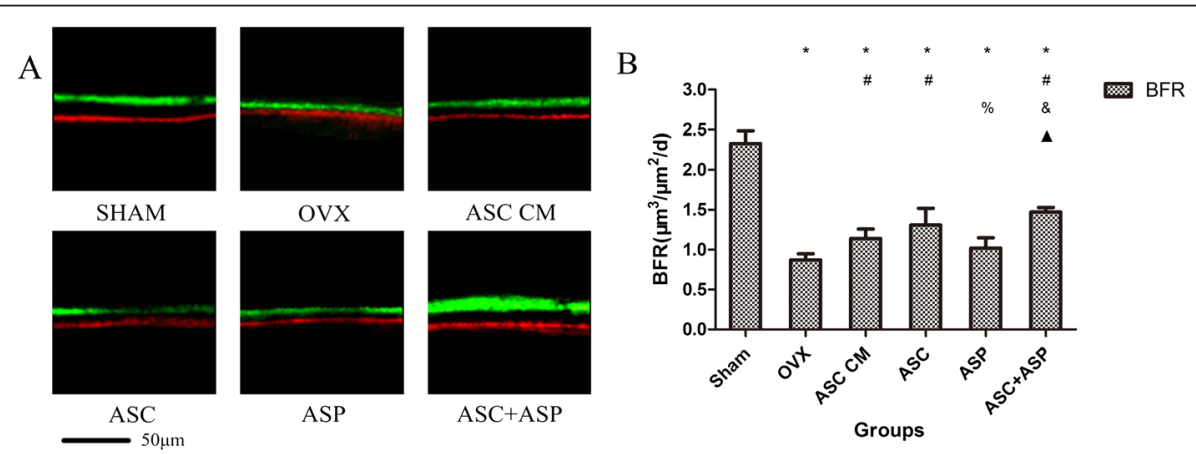

Fig. 2 Dynamic histomorphometric analyses of rat tibias. a Representative fluorescence images obtained from tibias after double labeling with alizarin red and calcein. Scale bar $=50 \mu \mathrm{m}$. b Bone formation rate (BFR) was measured from the tibia using Bio-quant software. Data are the mean \pm SD. ${ }^{*} p<0.05$ vs. Sham, \#p 0.05 vs. OVX, \&p<0.05 vs. ASC CM, $\% p<0.05$ vs. ASC, black triangle $p<0.05$ vs. ASP. OVX ovariectomized, ASC adipose-derived stromal cells, ASP aspirin 


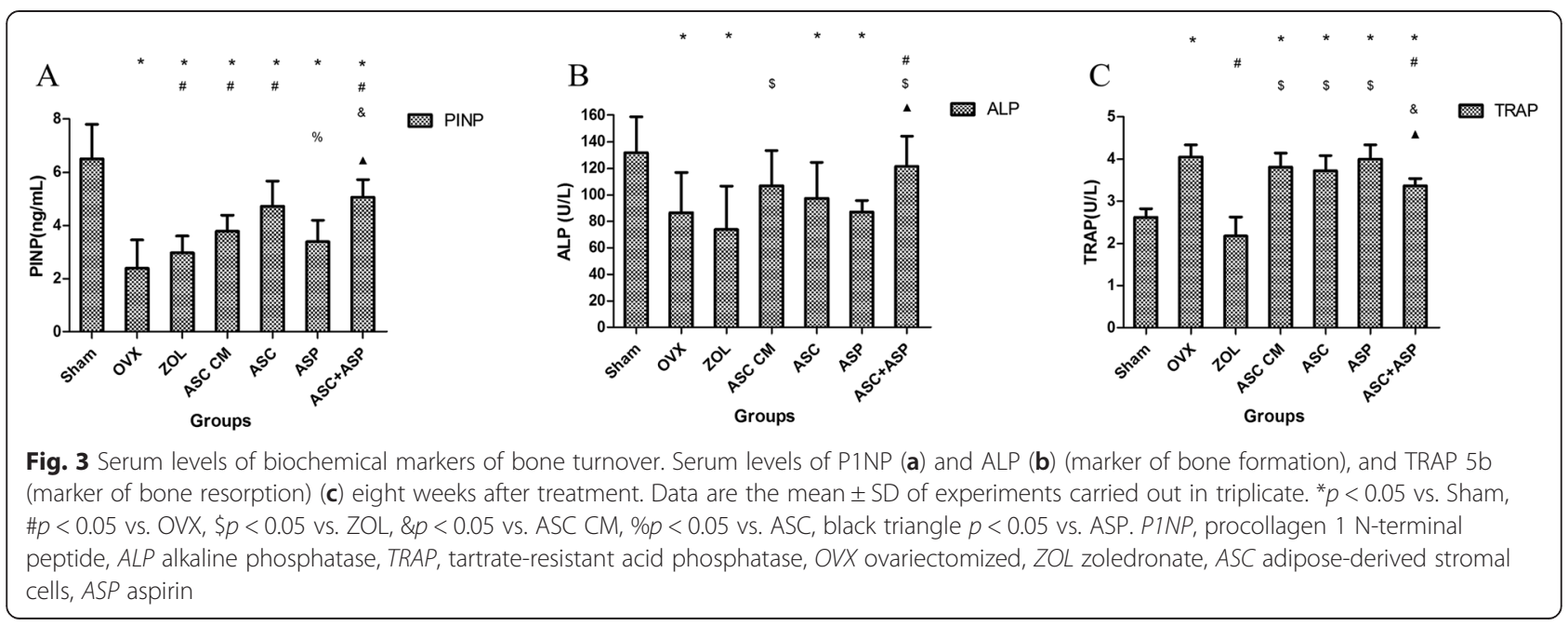

were reduced significantly compared with those in the OVX, ASC CM, and ASP groups (OVX group, $p=0.004$; ASC CM group, $p=0.038$; ASP group, $p=0.003$ ). However, there was no significant difference among the OVX, ASC CM, ASC, and ASP groups (Fig. 3c). These findings suggest that systemic administration of rASCs, ASC CM, or aspirin primarily affected bone formation and osteoblastic activity, and was also associated with bone resorption (especially in the ASC + ASP group).

\section{Serological assessment of calcium}

ELISAs suggested that serum levels of calcium in the OVX, ZOL, ASC CM, and ASP groups (OVX, ZOL, ASC CM groups, $p<0.001$; ASP group, $p=0.003$ ) decreased significantly compared with those in the sham group (Fig. 4). Calcium levels in the serum of rats in the ASC + ASP and ASC groups were comparable with

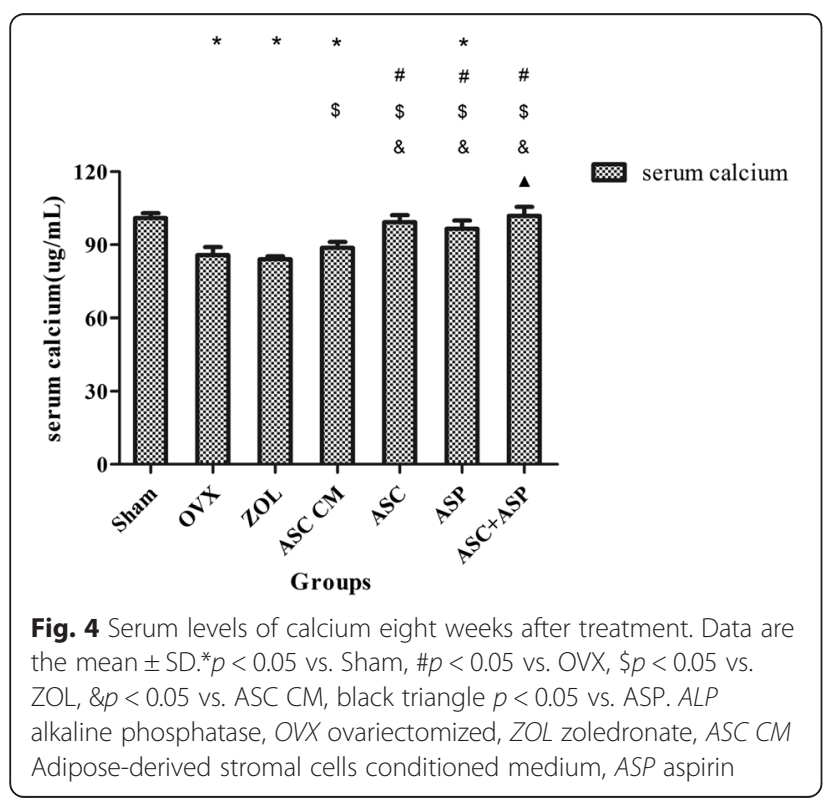

those of the sham group. Serum levels of calcium in the ASC + ASP group increased significantly compared with those in the OVX, ZOL, ASC CM, and ASP groups (OVX, ZOL, and ASC CM groups, $p<0.001$; ASP group, $p=0.002)$. However, serum levels of calcium decreased significantly in the ASC CM group compared with those in the other treatment groups (ASC, ASP, and ASC + ASP groups) $(p<0.001$, respectively).

\section{Serological assessment of pro-inflammatory cytokines (TNF- $\alpha$ and IFN- $\gamma$ )}

Serum levels of TNF- $\alpha$ and IFN- $\gamma$ among the seven groups are shown in Fig. 5. As expected, serum levels of TNF- $\alpha$ and IFN- $\gamma$ were increased significantly in the OVX, ZOL, ASC CM, and ASC groups (TNF- $\alpha$ : OVX and ASC CM groups, $p<0.001$; ZOL group, $p=0.001$; ASC group, $p=0.002$; IFN- $\gamma$ : OVX and ZOL groups, $p<0.001$; ASC CM group, $p=0.001$; ASC group $p=0.036$ ) compared with the sham group. Consistent with the results of bone microarchitecture, serum levels of TNF- $\alpha$ and IFN- $\gamma$ were decreased significantly in the ASC + ASP group compared with the OVX, ZOL, ASC CM, and ASC groups (TNF- $\alpha$ : OVX group, $p=0.001$; ZOL group, $p=$ 0.020 ; ASC CM group, $p=0.012$; ASC group, $p=0.038$; IFN- $\gamma$ : OVX and ZOL groups, $p<0.001$; ASC CM group, $p=0.001$; ASC group, $p=0.030$ ). Moreover, serum levels of TNF- $\alpha$ and IFN- $\gamma$ in the ASP group were significantly lower than those in the OVX, ZOL, and ASC CM groups (TNF- $\alpha$ : OVX group, $p=0.003$; ZOL group, $p=0.045$; ASC CM group, $p=0.032$; IFN$\gamma$ : OVX group, $p<0.001$; ZOL group, $p=0.001$; ASC CM group, $p=0.014$ ).

\section{Effect of aspirin on chemotactic ability in vitro}

The effect of aspirin on chemotactic ability in vitro is shown in Fig. 6. The ability of rASCs to migrate at aspirin concentrations of $0.001,0.01,0.1$, and $1 \mathrm{mM}$ was 

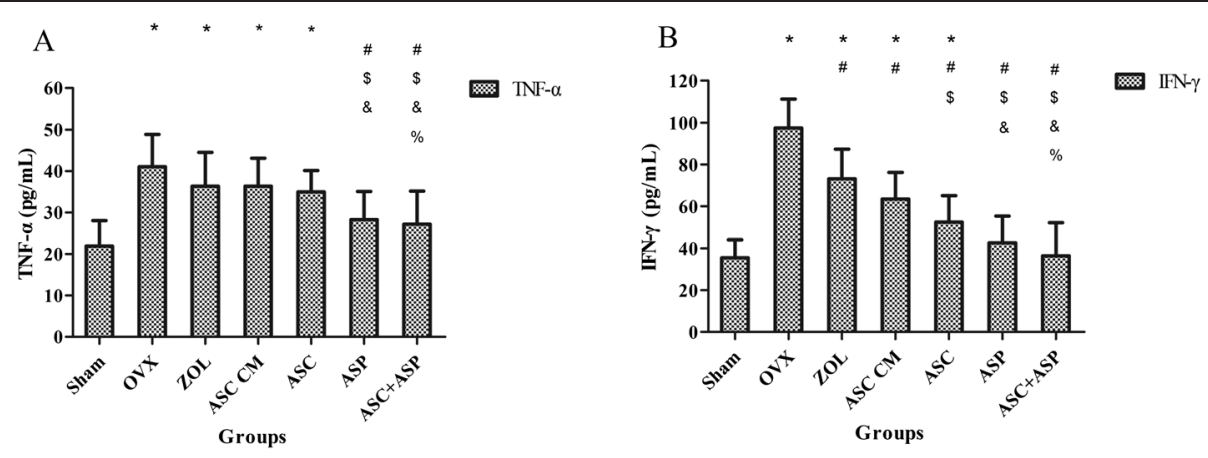

Fig. 5 Serum levels of TNF- $a$ and IFN- $\gamma$ by ELISA eight weeks after treatment. Serum levels of TNF- $\alpha(\mathbf{a})$ and IFN- $\gamma(\mathbf{b})$ among the seven groups. Data are the mean \pm SD of experiments undertaken in triplicate. ${ }^{*} p<0.05$ vs. Sham, $\# p<0.05$ vs. OVX, $\$ p<0.05$ vs. ZOL, $\& p<0.05$ vs. ASC CM, $\% p<0.05$ vs. ASC. OVX ovariectomized, ZOL zoledronate, ASC CM adipose-derived stromal cells conditioned medium

enhanced significantly compared with that in the control group $(0.001 \mathrm{mM}$ and $0.01 \mathrm{mM}, p<0.001 ; 0.1 \mathrm{mM}$, $p=0.016 ; 1 \mathrm{mM}, p=0.022$ ). At aspirin concentrations of $0.001 \mathrm{mM}$ and $0.01 \mathrm{mM}$, cell migration was $135 \%$ and $137 \%$ higher than that of the control group. Moreover, significant increases were observed in the 0.001$\mathrm{mM}$ and $0.01-\mathrm{mM}$ groups compared with $0.1-\mathrm{mM}$ (0.001 mM, $p=0.004 ; 0.01 \mathrm{mM}, p=0.003)$ and $1-\mathrm{mM}$ groups (0.001 mM, $p=0.003 ; 0.01 \mathrm{mM}, p=0.002)$. However, the cell-migration ratio in the $0.0001-\mathrm{mM}$ group was not increased significantly compared with that in the control group $(p>0.05)$. A significant difference between $0.1-\mathrm{mM}$ and $1-\mathrm{mM}$ groups was not observed.

\section{Effect of aspirin on homing of rASCs in vivo}

The effect of aspirin on homing of rASCs by celltrafficking assays in vivo is shown as a curve of the ratio of rASCs/bone marrow stromal cells (BMSCs) in the $\mathrm{BM}$ at different time points in Fig. 7. At 6, 12, and $24 \mathrm{~h}$ after treatment, the ratio of $\mathrm{rASCs} / \mathrm{BMSCs}$ in the OVX-ASP group was significantly greater than that in the OVX-Vehicle group $(33.77 \pm 3.49 \%$ vs. $26.9 \pm 1.56 \%$ at $6 \mathrm{~h}, p=0.036 ; 60.37 \% \pm 0.78 \%$ vs. $49.7 \% \pm 5.32 \%$ at $12 \mathrm{~h}, p=0.027 ; 63.27 \% \pm 4.11 \%$ vs. $49.9 \% \pm 6.58 \%$ at $24 \mathrm{~h}, p=0.041)$. The difference in the ratio of homing of rASCs increased gradually from $6 \mathrm{~h}$ to $24 \mathrm{~h}$ between the OVX-ASP group and OVX-Vehicle group, and reached a maximum at $24 \mathrm{~h}$. At $48 \mathrm{~h}$ and $72 \mathrm{~h}$, the ratio of rASCs/ BMSCs in the OVX-ASP group was higher than that in the OVX-Vehicle group, but this difference was not significant. In the OVX-ASP group, the ratio of rASCs/ BMSCs increased rapidly before $24 \mathrm{~h}$ after treatment and gradually declined until $72 \mathrm{~h}$.

\section{Discussion}

In the present study, we demonstrated for the first time that the co-administration of aspirin and allogeneic rASCs partially prevented OVX-induced bone loss in OVX rats. We also confirmed that the effects of co-administered aspirin and allogeneic rASCs can attenuate bone loss more effectively than either one alone. Nevertheless, the single administration of aspirin or allogeneic rASCs (and even rASCs CM) also partially prevented bone loss in

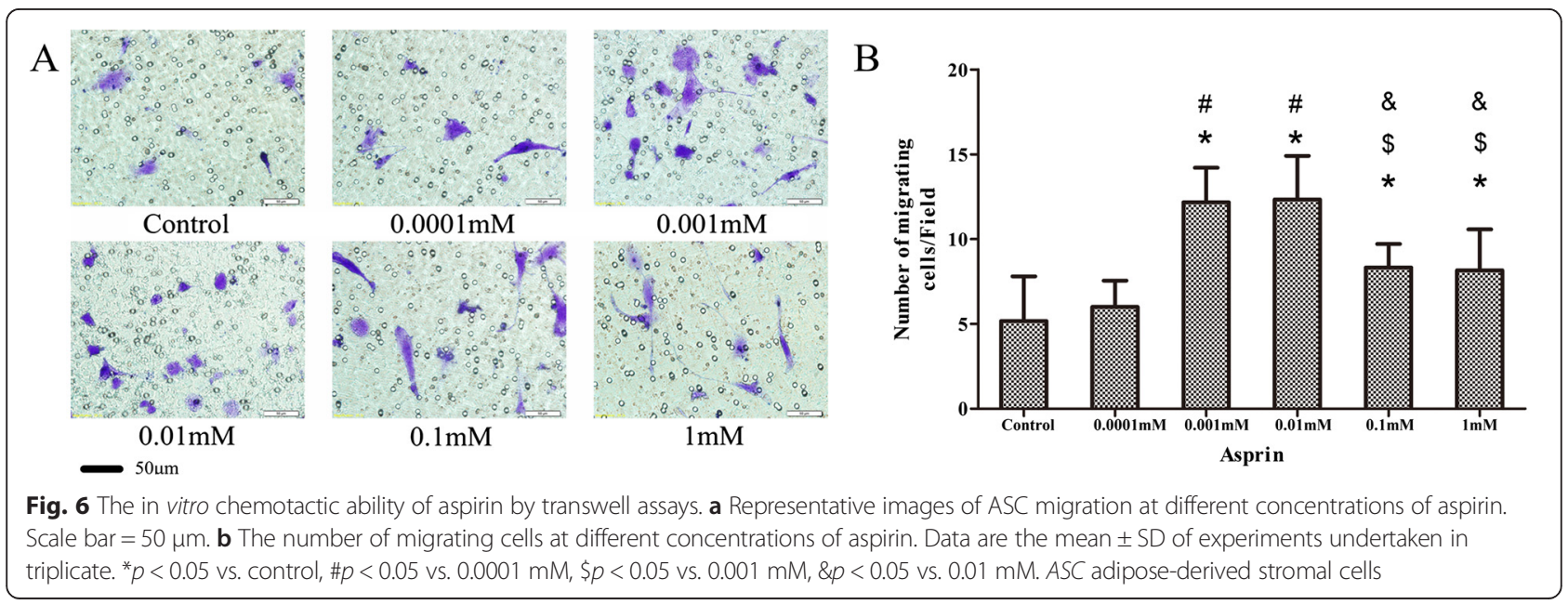




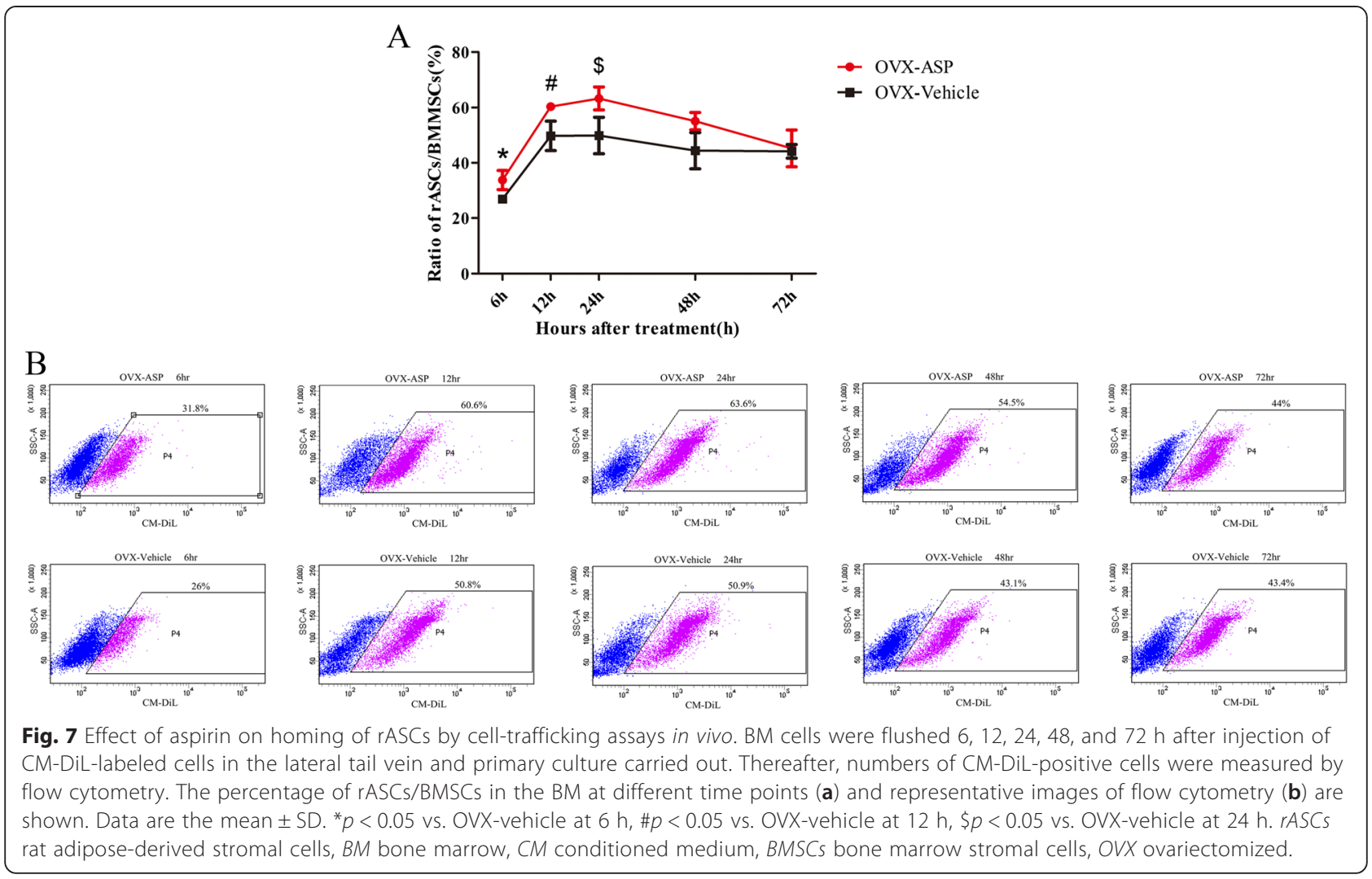

comparison with the OVX control group. To fully explore the underlying mechanism of this co-operation, a combination of in vitro and in vivo experiments was carried out.

We found that aspirin decreased serum levels of the pro-inflammatory cytokines TNF- $\alpha$ and IFN- $\gamma$ markedly. Also, aspirin enhanced the chemotactic ability of rASCs in vitro and accelerated homing of rASCs into BM in vivo. These results suggest that aspirin has comprehensive anti-inflammatory effects and promotes homing of rASCs, which might be associated with attenuating OVX-induced bone loss.

\section{Anti-inflammatory effect of aspirin for bone regeneration}

We found that aspirin significantly suppressed serum levels of the pro-inflammatory cytokines TNF- $\alpha$ and IFN$\gamma$, which is in accordance with the findings of previously reported studies [28, 37]. Meanwhile, the aspirin groups (ASP and ASC + ASP groups) showed partially prevented bone loss in OVX rats. Hence, we speculated that TNF- $\alpha$ and IFN- $\gamma$ may play a role in bone metabolism.

Firstly, several recent lines of evidence have supported the hypothesis that IFN- $\gamma$ or TNF- $\alpha$ accelerated osteoclastogenesis through T-cell immunity [38-41]. Studies have shown that TNF- $\alpha$ (which is derived from activated $\mathrm{T}$ cells as a result of OVX) augments macrophage colony-stimulating factor (M-CSF)-induced and receptor activator of nuclear factor-kappa B ligand (RANKL)-induced osteoclastogenesis via the TNF- $\alpha$ receptor p55 when estrogen deficiency induces bone loss [38, 39]. Cenci et al. discovered that IFN- $\gamma$ enhances by the upregulated expression of class-II transactivator located in antigen-presenting cells (APC) and $\mathrm{T}$ lymphocytes in OVX mice, resulting in enhanced antigen presentation by macrophages, as well as enhanced $\mathrm{T}$ cell activation and lifespan [40]. In this research, the serum levels of TNF- $\alpha$ and IFN- $\gamma$ in the OVX group were highest among all seven groups, which also supports the abovementioned hypothesis. In addition, Gao et al. demonstrated that IFN- $\gamma$ indirectly promotes bone resorption by multiple pathways ( $\mathrm{T}$ cell activation and $\mathrm{T}$ cell secretion of RANKL and TNF- $\alpha$ ), but blunts osteoclast formation through the direct targeting of osteoclast precursors. However, under conditions of estrogen deficiency, infection and inflammation, the net balance of these two opposing factors is biased toward bone resorption [41]. In summary, co-operation of TNF- $\alpha$ and IFN- $\gamma$ enhances the proliferation and activation of $\mathrm{T}$ cells, and further augments M-CSF-induced and RANKL-induced osteoclastogenesis. Our findings have demonstrated that the levels of TRAP, a marker of bone resorption, in the ASC + ASP group significantly decreased compared with those in the OVX group, which is consistent with the results of 
the above-mentioned studies. However, the level of TRAP in the ASP group only reduced slightly compared with the OVX group. This finding appeared to be inconsistent with the observation that TNF- $\alpha$ and IFN- $\gamma$ were suppressed by aspirin. We conjectured that perhaps a small amount of DMSO (1 \% DMSO for aspirin solubilization) promoted osteoclast differentiation [42] in the OVX model and attenuated the inhibition of osteoclastogenesis. In addition, Zhang et al. [43] showed that aspirin inhibits osteoclast differentiation and bone resorption in a dosedependent manner, whereby low-dose aspirin has a weak effect on osteoclast inhibition. In this study, we only administered low-dose aspirin to the rats, equivalent to $15.87 \mathrm{mg} / \mathrm{kg} / \mathrm{d}$ aspirin for humans, as calculated by the relative body surface area between rats and humans [44]. Therefore, we determined that aspirin alone did not significantly decrease TRAP levels compared to the OVX groups as a probable consequence of the combination of low-dose aspirin and DMSO.

Secondly, TNF- $\alpha$ and IFN- $\gamma$ promoted the apoptosis of BMMSCs and osteoblasts. Liu et al. demonstrated that TNF- $\alpha$ and IFN- $\gamma$ synergistically enhance BMMSC apoptosis by the inhibition of the TNFR2/NFkB pathway and Fas internalization, and inhibit BMMSC-mediated bone formation [28]. More recently, Wang et al. showed that TNF- $\alpha$ and IFN- $\gamma$ synergistically downregulate expression of FAS ligand in osteoblast progenitors and reduce the ability to induce osteoclast apoptosis by osteoblasts [45].

Taken together, the above-described studies and the results from the present study suggest that aspirin suppressed levels of pro-inflammatory cytokines, such as TNF- $\alpha$ and IFN- $\gamma$, and partially prevented bone loss, which may be associated with decreasing osteoclastogenesis and inhibiting the apoptosis of BMMSCs and osteoblasts. The mechanisms involved in aspirin and bone loss will be the subject of future studies.

\section{Chemotactic effects of aspirin on bone regeneration in vitro and in vivo}

The original intention of the present study was to use aspirin as an anti-inflammatory agent to attenuate bone loss in OVX rats. Interestingly, we found that aspirin (0.001 mM-1 mM) increased the migration of rASCs in vitro. Reports of the effects of aspirin on the migration and homing of adipose-derived stem cells are lacking. $\mathrm{Hu}$ et al. reported that low-dose aspirin promoted the migration and adhesion of endothelial progenitor cells due to the vasculoprotective benefits of aspirin [33]. The results of the present study are consistent with those studies. However, Chen et al. hypothesized that aspirin decreases the proliferative, migratory, and adhesive effects of endothelial progenitor cells at higher concentrations $(1 \mathrm{mM}-10 \mathrm{mM})$ [46]. These divergent views might have resulted from the aspirin dose, variations in type and/or species of cells, as well as the in vitro or in vivo assays we employed.

Also, Brunelli et al. reported that HCT 1026 (an agent with nonsteroidal anti-inflammatory activity) significantly enhanced the engrafting to muscle of mesoangioblasts, a type of stem cell shown to be effective in correcting genetic defects of muscle. Interestingly, the increased homing of mesoangioblasts to muscle has been shown to be accompanied by a concomitant reduction of their number in the relevant filter organs (liver, lung, kidney, and spleen) [34]. These findings regarding enhanced stem cell homing by a nonsteroidal anti-inflammatory agent in vivo are similar to our current results, although the underlying mechanisms have not yet been explained. Although we have not provided histological evidence as to why aspirin promoted homing to the BM rather than elsewhere, the in vivo and in vitro cell migration induced by aspirin detected by both FCM and the transwell assays validated this finding. It may be that the ability of aspirin to induce cell migration is not specific for bone, and other organs or tissues may also benefit. However, the bone will indeed benefit if the aspirin induces rASCs to home to bone. In future studies, we will use more specific methods to investigate the mechanism of stem cell homing.

\section{Osteogenesis upon co-administration of aspirin and allo- geneic ASCs in OVX rats}

The single administration of aspirin, allogeneic rASCs or rASCs CM resulted in partial osteogenesis. However, co-administration of aspirin and allogeneic ASCs had better effects upon bone regeneration. With regard to the co-administration effects of aspirin and rASCs, aspirin improved the osteogenic microenvironment of rASCs by suppressing expression of pro-inflammatory cytokines and accelerating homing of rASCs, but allogeneic rASCs constitute an abundant source of stem cells for therapeutic use and have excellent immunosuppressive and immunomodulatory properties [47, 48]. In accordance with our conclusions, Liu Y et al. reported that implantation of BMMSCs/hydrogel/aspirin resulted in improvement of bone generation in calvarial defects [28]. Taken together, the co-administration of aspirin and stem cells appears to be a feasible strategy to promote stem cell-mediated bone regeneration. However, young rASCs were administered in this research, which have better osteogenic effects than aged rASCs [49]. Moreover, the more long-term osteogenic effects of young rASCs were not studied. Next, we will study the long-term osteogenic effects of administering young rASCs. Recently, Yan et al. [50] reported that 5-azacytidine improves the osteogenic differentiation potential of aged ASCs by DNA demethylation, which may indicate a useful area of further investigation. 
Interestingly, we observed that the $\mathrm{CM}$ of allogeneic rASCs also partially prevented OVX-induced bone loss in rats and resulted in a higher BMD than that in the ASC + ASP group. In support of our hypothesis, Mirsaidi et al. showed that the osteogenic potential of human BMSCs cultured with homogeneous ASC-CM dramatically increased compared with that of BMSCs cultured with BMSC-CM [51]. Sun et al. discovered that in vitro, treatment with ASC-CM enhanced osteogenic differentiation in stromal cells and pre-osteoblasts [52]. Jee et al. demonstrated that the $\mathrm{CM}$ of human umbilical cord blood-derived mesenchymal stem cells preserved BMD and improved trabecular parameters comparable with that of the OVX group and appeared to be mediated by a paracrine mechanism rather than direct engraftment of MSCs [6]. Moreover, Banas et al. reported that MSCs from adipose tissue produced significantly more immunosuppressive IL-1RA than BMMSCs; also IL-1RA was a robust anti-inflammatory cytokine and inhibited the binding of IL- $1 \alpha$ and IL-1 [48].

\section{Conclusions}

We demonstrated that the systematic administration of aspirin and allogeneic ASCs attenuated bone loss more effectively than either one alone in OVX rats. Also, aspirin plays a key part in bone regeneration owing to its anti-inflammatory and chemotactic abilities. The results of our study will shed more light on the potential use of aspirin and allogeneic ASCs in the prevention/treatment of osteoporosis.

\begin{abstract}
Abbreviations
ALP: alkaline phosphatase; ASP: Aspirin; ASCs: Adipose-derived stromal cells; BFR: Bone formation rate; BM: Bone marrow; BMMSCs: Bone marrow-derived mesenchymal stem cells; BMSCs: Bone marrow stromal cells;

CM: Conditioned medium; ELISA: Enzyme-linked immunosorbent assay; IFN: interferon; MSCs: Mesenchymal stem cells; P1NP: Procollagen $1 \mathrm{~N}$ terminal peptide; RANKL: Nuclear factor-kappa B ligand; TNF: tumor necrosis factor; TRAP: Tartrate-resistant acid phosphatase; ZOL: zoledronate.
\end{abstract}

\section{Competing interests}

The authors declare that they have no competing interests.

\section{Authors' contributions}

$\mathrm{HL}$ carried out the animal experiments, micro-CT, and drafted the manuscript. WL carried out the slicing of undecalcified bones, dynamic histomorphometric analyses, and revised the manuscript. YSL participated in the design of the study, performed the statistical analysis, and helped to revise the manuscript. XZ carried out cell culture experiments and partially drafted the manuscript. YSZ conceived of the study and participated in its design, as well as manuscript writing and revision. All authors read and approved the final manuscript.

\section{Acknowledgments}

We thank the National Center for Protein Sciences Beijing (Peking University) for assistance with flow cytometry analysis. We also thank Dr. Zhonglin Fu, Dr. Longwei LV, and Mrs. Tong Chen for his/her help with data collection of flow cytometry, cell-trafficking experiment, and serological assays, respectively. This work was supported by grants from the National Natural Science Foundation of China (No. 81170937) and the Program for New Century Excellent Talents in University from Ministry of Education (NCET-11-0026).

\section{Author details}

${ }^{1}$ The Central Laboratory, Peking University School and Hospital of Stomatology, Beijing 100081, China. ${ }^{2}$ Department of Prosthodontics, Peking University School and Hospital of Stomatology, 22 Zhongguancun South Avenue, Haidian District, Beijing 100081, China. ${ }^{3}$ National Engineering Lab for Digital and Material Technology of Stomatology, Peking University School and Hospital of Stomatology, Beijing 100081, China.

Received: 21 May 2015 Revised: 20 August 2015

Accepted: 1 October 2015 Published online: 16 October 2015

\section{References}

1. Burge R, Dawson-Hughes B, Solomon DH, Wong JB, King A, Tosteson A. Incidence and economic burden of osteoporosis-related fractures in the United States, 2005-2025. J Bone Miner Res. 2007;22:465-75.

2. Silva BC, Bilezikian JP. New approaches to the treatment of osteoporosis. Annu Rev Med. 2011;62:307-22.

3. Iwamoto J, Seki A, Sato Y. Effect of combined teriparatide and monthly minodronic acid therapy on cancellous bone mass in ovariectomized rats: a bone histomorphometry study. Bone. 2014;64:88-94.

4. Mulgund M, Beattie KA, Wong AK, Papaioannou A, Adachi JD. Assessing adherence to teriparatide therapy, causes of nonadherence and effect of adherence on bone mineral density measurements in osteoporotic patients at high risk for fracture. Ther Adv Musculoskelet Dis. 2009;1:5-11.

5. Body JJ, Bergmann P, Boonen S, Boutsen Y, Devogelaer JP, Goemaere S, et al. Evidence-based guidelines for the pharmacological treatment of postmenopausal osteoporosis: a consensus document by the Belgian Bone Club. Osteoporos Int. 2010;21:1657-80.

6. An JH, Park H, Song JA, Ki KH, Yang JY, Choi HJ, et al. Transplantation of human umbilical cord blood-derived mesenchymal stem cells or their conditioned medium prevents bone loss in ovariectomized nude mice. Tissue Eng Part A. 2013;19:685-96.

7. Miller PD, McCarthy EF. Bisphosphonate-associated atypical sub-trochanteric femur fractures: paired bone biopsy quantitative histomorphometry before and after teriparatide administration. Semin Arthritis Rheum. 2015;44:477-82.

8. Liu X, Wang JA, Ji XY, YU SP, Wei L. Preconditioning of bone marrow mesenchymal stem cells by prolyl hydroxylase inhibition enhances cell survival and angiogenesis in vitro and after transplantation into the ischemic heart of rats. Stem Cell Res Ther. 2014;5:111.

9. Qayyum AA, Mathiasen AB, Kastrup J. Stem cell therapy to treat heart ischaemia: implications for diabetes cardiovascular complications. Curr Diab Rep. 2014;14:554.

10. Zhou F, Gao S, Sun C, Cao L, Ren H, Li Q, et al. Adipose-derived stem cells in stroke treatment: translational possibility and mechanism. Chin Med J (Engl). 2014;127:3657-63.

11. Boninger ML, Wechsler LR, Stein J. Robotics, stem cells, and brain-computer interfaces in rehabilitation and recovery from stroke: updates and advances. Am J Phys Med Rehabil. 2014;93:S145-54.

12. Ledford H. Stem-cell success aids diabetes fight. Nature. 2014;514:281.

13. Vogel G. Biomedicine. Stem cell recipe offers diabetes hope. Science. 2014;346:148.

14. Ahmed TA, Hincke MT. Mesenchymal stem cell-based tissue engineering strategies for repair of articular cartilage. Histol Histopathol. 2014;29:669-89.

15. Kumar S, Wan C, Ramaswamy G, Clemens TL, Ponnazhagan S. Mesenchymal stem cells expressing osteogenic and angiogenic factors synergistically enhance bone formation in a mouse model of segmental bone defect. Mol Ther. 2010;18:1026-34.

16. Zhao M, Zhou J, Li X, Fang T, Dai W, Yin W, et al. Repair of bone defect with vascularized tissue engineered bone graft seeded with mesenchymal stem cells in rabbits. Microsurgery. 2011;31:130-7.

17. Li Y, Charif N, Mainard D, Bensoussan D, Stoltz JF, de Isla N. Donor's age dependent proliferation decrease of human bone marrow mesenchymal stem cells is linked to diminished clonogenicity. Biomed Mater Eng. 2014;24:47-52

18. Coelho BP, Giraldi-Guimaraes A. Effect of age and gender on recovery after stroke in rats treated with bone marrow mononuclear cells. Neurosci Res. 2014;88:67-73.

19. Valyushina MP, Buravkova LB. Age-related differences in rat multipotent mesenchymal stromal bone marrow cells. Bull Exp Biol Med. 2013;155:129-33. 
20. Gimble JM, Nuttall ME. Adipose-derived stromal/stem cells (ASC) in regenerative medicine: pharmaceutical applications. Curr Pharm Des. 2011;17:332-9

21. Baer PC, Geiger H. Adipose-derived mesenchymal stromal/stem cells: tissue localization, characterization, and heterogeneity. Stem Cells Int. 2012;2012:812693.

22. Strong AL, Ohlstein JF, Jiang Q, Zhang Q, Zheng S, Boue SM, et al. Novel daidzein analogs enhance osteogenic activity of bone marrow-derived mesenchymal stem cells and adipose-derived stromal/stem cells through estrogen receptor dependent and independent mechanisms. Stem Cell Res Ther. 2014,5:105

23. Carvalho PP, Gimble JM, Dias IR, Gomes ME, Reis RL. Xenofree enzymatic products for the isolation of human adipose-derived stromal/stem cells. Tissue Eng Part C Methods. 2013;19:473-8.

24. Liu HY, Chiou JF, Wu AT, Tsai CY, Leu JD, Ting LL, et al. The effect of diminished osteogenic signals on reduced osteoporosis recovery in aged mice and the potential therapeutic use of adipose-derived stem cells. Biomaterials. 2012;33:6105-12.

25. Mohammadzadeh A, Pourfathollah AA, Shahrokhi S, Hashemi SM, Moradi SL, Soleimani M. Immunomodulatory effects of adipose-derived mesenchymal stem cells on the gene expression of major transcription factors of T cell subsets. Int Immunopharmacol. 2014;20:316-21.

26. Wang $X$, Liu C, Li S, Xu Y, Chen P, Liu Y, et al. Effects of continuous passage on immunomodulatory properties of human adipose-derived stem cells. Cell Tissue Bank. 2015;16:143-50.

27. Carbone LD, Tylavsky FA, Cauley JA, Harris TB, Lang TF, Bauer DC, et al. Association between bone mineral density and the use of nonsteroidal antiinflammatory drugs and aspirin: impact of cyclooxygenase selectivity. J Bone Miner Res. 2003;18:1795-802.

28. Liu Y, Wang L, Kikuiri T, Akiyama K, Chen C, Xu X, et al. Mesenchymal stem cell-based tissue regeneration is governed by recipient $T$ lymphocytes via IFN-gamma and TNF-alpha. Nat Med. 2011;17:1594-601.

29. Kwon MS, Shim EJ, Seo YJ, Choi SS, Lee JY, Lee HK, et al. Effect of aspirin and acetaminophen on proinflammatory cytokine-induced pain behavior in mice. Pharmacology. 2005;74:152-6.

30. Yamaza T, Miura Y, Bi Y, Liu Y, Akiyama K, Sonoyama W, et al. Pharmacologic stem cell based intervention as a new approach to osteoporosis treatment in rodents. PLoS One. 2008;3, e2615.

31. Buckland M, Jago CB, Fazekasova H, Scott K, Tan PH, George AJ, et al. Aspirin-treated human DCs up-regulate ILT-3 and induce hyporesponsiveness and regulatory activity in responder T cells. Am J Transplant. 2006;6:2046-59.

32. Shi S, Gronthos S, Chen S, Reddi A, Counter CM, Robey PG, et al. Bone formation by human postnatal bone marrow stromal stem cells is enhanced by telomerase expression. Nat Biotechnol. 2002;20:587-91.

33. Hu Z, Zhang F, Yang Z, Zhang J, Zhang D, Yang N, et al. Low-dose aspirin promotes endothelial progenitor cell migration and adhesion and prevents senescence. Cell Biol Int. 2008;32:761-8.

34. Brunelli S, Sciorati C, D'Antona G, Innocenzi A, Covarello D, Galvez BG, et al. Nitric oxide release combined with nonsteroidal antiinflammatory activity prevents muscular dystrophy pathology and enhances stem cell therapy. Proc Natl Acad Sci U S A. 2007;104:264-9.

35. Bouxsein ML, Boyd SK, Christiansen BA, Guldberg RE, Jepsen KJ, Müller R. Guidelines for assessment of bone microstructure in rodents using micro-computed tomography. J Bone Miner Res. 2010;25:1468-86.

36. Liu YS, OU ME, Liu H, Gu M, Lv LW, Fan C, et al. The effect of simvastatin on chemotactic capability of SDF-1alpha and the promotion of bone regeneration. Biomaterials. 2014;35:4489-98.

37. Wang L, Zhao Y, Liu Y, Akiyama K, Chen C, Qu C, et al. IFN- $\gamma$ and TNF-a synergistically induce mesenchymal stem cell impairment and tumorigenesis via NFKB signaling. Stem Cells. 2013;31:1383-95.

38. Cenci S, Weitzmann MN, Roggia C, Namba N, Novack D, Woodring J, et al. Estrogen deficiency induces bone loss by enhancing T-cell production of TNF-alpha. J Clin Invest. 2000;106:1229-37.

39. Roggia C, Gao Y, Cenci S, Weitzmann MN, Toraldo G, Isaia G, et al. Upregulation of TNF-producing $T$ cells in the bone marrow: a key mechanism by which estrogen deficiency induces bone loss in vivo. Proc Natl Acad Sci U S A. 2001;98:13960-5.

40. Cenci S, Toraldo G, Weitzmann MN, Roggia C, Gao Y, Qian WP, et al. Estrogen deficiency induces bone loss by increasing $T$ cell proliferation and lifespan through IFN-gamma-induced class II transactivator. Proc Natl Acad Sci U S A. 2003;100:10405-10.

41. Gao Y, Grassi F, Ryan MR, Terauchi M, Page K, Yang X, et al. IFN-gamma stimulates osteoclast formation and bone loss in vivo via antigen-driven $T$ cell activation. J Clin Invest. 2007;117:122-32.

42. Lemieux JM, Wu G, Morgan JA, Kacena MA. DMSO regulates osteoclast development in vitro. In Vitro Cell Dev Biol Anim. 2011;47:260-7.

43. Zhang Y, Niu E, Li Y, Liang Y, Qin J, Yi H. Effect of aspirin on rat osteoclast differentiation and bone resorption activity. Chin J Osteoporos. 2013;19:435-40.

44. Zhang Y. Pharmacology experiment. 2nd ed. Beijing: People's Medical Publishing House; 1996.

45. Wang L, Liu S, Zhao Y, Liu D, Liu Y, Chen C, et al. Osteoblast-induced osteoclast apoptosis by fas ligand/FAS pathway is required for maintenance of bone mass. Cell Death Differ. 2015;22:1654-64.

46. Chen TG, Chen JZ, Xie XD. Effects of aspirin on number, activity and inducible nitric oxide synthase of endothelial progenitor cells from peripheral blood. Acta Pharmacol Sin. 2006;27:430-6.

47. Seki A, Sakai Y, Komura T, Nasti A, Yoshida K, Higashimoto M, et al. Adipose tissue-derived stem cells as a regenerative therapy for a mouse steatohepatitis-induced cirrhosis model. Hepatology. 2013;58:1133-42.

48. Banas A, Teratani T, Yamamoto Y, Tokuhara M, Takeshita F, Osaki M, et al. IFATS collection: in vivo therapeutic potential of human adipose tissue mesenchymal stem cells after transplantation into mice with liver injury. Stem Cells. 2008:26:2705-12.

49. Girolamo L, Lopa S, Arrigoni E, Sartori MF, Baruffaldi Preis FW, Brini AT. Human adipose-derived stem cells isolated from young and elderly women: their differentiation potential and scaffold interaction during in vitro osteoblastic differentiation. Cytotherapy. 2009;11:793-803.

50. Yan X, Ehnert S, Culmes M, Bachmann A, Seeliger C, Schyschka L, et al. 5azacytidine improves the osteogenic differentiation potential of aged human adipose-derived mesenchymal stem cells by DNA demethylation. PLoS One. 2014;9, e90846.

51. Mirsaidi A, Genelin K, Vetsch JR, Stanger S, Theiss F, Lindtner RA, et al. Therapeutic potential of adipose-derived stromal cells in age-related osteoporosis. Biomaterials. 2014;35:7326-35.

52. Cho SW, Sun HJ, Yang JY, Jung JY, Choi HJ, An JH, et al. Human adipose tissue-derived stromal cell therapy prevents bone loss in ovariectomized nude mouse. Tissue Eng Part A. 2012;18:1067-78.

\section{Submit your next manuscript to BioMed Central and take full advantage of:}

- Convenient online submission

- Thorough peer review

- No space constraints or color figure charges

- Immediate publication on acceptance

- Inclusion in PubMed, CAS, Scopus and Google Scholar

- Research which is freely available for redistribution 